\title{
Semi-quantitative evaluation of signal intensity and contrast-enhancement in Modic changes
}

\author{
Marta Tibiletti ${ }^{1}$, Cristina Ciavarro², Vlasta Bari ${ }^{3}$, lain W. McCall ${ }^{4}$, Jill P. G. Urban5, Marco Brayda-Bruno ${ }^{6}$ \\ and Fabio Galbusera ${ }^{2^{*}}$ (i)
}

\begin{abstract}
Background: Semi-quantitative evaluation of Modic changes (MCs) has recently been proposed as a way to standardise and increase repeatability of clinical studies. This study is aimed at developing semi-quantitative measures of enhancement, given by contrast agent injection, on T1-weighted images in MCs, and to investigate their reliability and relation with MC types.

Methods: Thirty-seven subjects suffering from low back pain underwent T1-weighted and T2-weighted turbo spin-echo sequences. Five minutes after the injection of a paramagnetic contrast agent, a second T1-weighted sequence was acquired. Regions of interest (ROIs) corresponding to MCs were selected manually on the unenhanced image; control ROls in the "healthy" bone marrow were selected. For each ROI, the mean signal intensity (SI) of unenhanced pixels and the mean absolute and normalised difference in SI between unenhanced and contrast-enhanced pixels values were calculated.

Results: A total of 103 MCs were recognised and 61 were semi-quantitatively analysed: 16 type I, 34 type II and 11 type I/II. Regarding controls, MCs I showed a lower SI on the unenhanced T1-weighted images and a marked contrast enhancement (CE); MCS II showed a higher SI than controls on unenhanced images and a lower or comparable CE; and MCS I/II presented an intermediate SI on the unenhanced images and a marked CE. Inter-rater and intra-rater agreements were found to be excellent or substantial.

Conclusions: Semi-quantitative measurements could differentiate MC types in terms of unenhanced SI and of CE with respect to "healthy" bone marrow.
\end{abstract}

Keywords: Modic changes, Gadolinium-based contrast agent, Contrast enhancement, Magnetic resonance imaging, Spine

\section{Key points}

- Contrast enhancement differed between Modic changes and healthy bone marrow.

- Type I Modic changes showed a marked contrast enhancement.

- Type II Modic changes showed a low contrast enhancement.

\section{Introduction}

Modic changes (MCs) are common variations of signal intensity in the endplate and vertebral body seen on magnetic resonance imaging (MRI) [1,2]. Depending on their characteristics on T1-weighted and T2-weighted images, MCs are classified into type I (hypointense on $\mathrm{T} 1$ images and hyperintense on T2 images), type II (hyperintense on both T1-weighted and T2-weighted images) and type III (hypointense in both T1-weighted and T2-weighted images). A normal endplate is considered grade 0 , and mixed types can co-exist in the same endplate [3]. MC types can change with time, but no fixed evolution pattern has been recognised [4]. MCs

* Correspondence: fabio.galbusera@grupposandonato.it

${ }^{2}$ IRCCS Galeazzi Orthopedic Institute, Via Galeazzi 4, 20161 Milan, Italy

Full list of author information is available at the end of the article 
I are thought to represent an ongoing active degenerative process demonstrated by fissured endplates with adjacent vascular granulation tissue within the bone marrow [1]. MCs II are considered signs of fatty marrow degeneration [1]: fatty marrow, which consists mainly of fat cells, is characterised by sparse vascularisation [5]. MCs I commonly progress to MCs II, and mixed-type MCs I/II are observed frequently [3,6]. Although MCs II are believed to be more stable [1], transition to MCs I has also been documented [7].

The relationship between low back pain (LBP) and MCs is controversial, although it has been suggested that MCs could help to classify patients suffering from nonspecific LBP [8] and could influence the outcome of surgical treatment [9-12]. Conflicting results have been obtained in different studies, possibly in part due to variations in study methodology and $\mathrm{MC}$ diagnosis. In most MC studies to date, evaluations are made with the naked eye and are thus subjective [13]. A semi-quantitative evaluation of MCs may be helpful in investigating the relationship between MCs, clinical signs of LBP and surgical outcome.

Although MCs are defined as signal intensity abnormalities, only two studies have recently proposed semiquantitative analysis of their signal intensity [13, 14]. Wang et al. [13] in particular proposed semi-quantitative measures on T1-weighted and T2-weighted images and showed the reliability of such methods.

In the case of suspected tumours, infections and vascular malformations [15], spinal MRI examinations are often accompanied by the intravenous injection of a contrast agent, a gadolinium chelate; this is not the case for LBP, for which a contrast injection is not commonly executed. Because the contrast agent shortens the $\mathrm{T} 1$ relaxation time, the tissues where the contrast agent pools (typically vessels, hyperaemic tissues and joint spaces) result in a higher signal on contrast-enhanced T1-weighted images compared with unenhanced T1-weighted images [15], in proportion to the concentration of the contrast agent [16].

Given the different vascular characteristics of MCs, the aim of the present study was to evaluate the contrast enhancement of MCs on T1-weighted images, hypothesising differences among the different types of MCs, by means of a semi-quantitative analysis.

\section{Methods}

\section{Population}

Thirty-seven patients affected by LBP were enrolled prospectively in this study, in which the primary outcome was to study contrast diffusion in intervertebral discs [17]. The selected population included male and female subjects, with an age of $42.5 \pm 9.1$ years (mean \pm standard deviation) and an age range from 18 to 60 years. Each patient received detailed information regarding the study protocol and gave her/his consent. Exclusion criteria were: age under 18 or over 60 years, contrast agent allergy, reduced renal function, and contraindications to MRI. The study was approved by the local ethical committee.

\section{Radiological evaluation}

MRI of the lumbar spine was performed with a 1.5-T scanner (Avanto; Siemens, Erlangen, Germany) with a phased-array back coil. Standard examinations included routine sagittal and axial T1-weighted (repetition time $=500 \mathrm{~ms}$, echo time $=13 \mathrm{~ms}$ ) and T2weighted (repetition time $=4180 \mathrm{~ms}$, echo time $=104$ $\mathrm{ms})$ turbo spin-echo sequences as well as axial T2weighted sequences. In addition, ProHance (gadoteridol; Bracco Diagnostics, Princeton, RI, USA), a paramagnetic macrocyclic non-ionic contrast agent, was injected at a dose of $0.2 \mathrm{mmol} / \mathrm{kg}$ and a second T1weighted image was taken approximately 5 minutes after contrast injection. A higher dose with respect to standard clinical applications was used following the indications of a previous study about diffusion in the intervertebral disc [18].

A musculoskeletal radiologist with more than 30 years of experience noted the presence and the type of MCs in the endplates from T12-L1 to L5-S1. Only the MCs with a vertical height of more than $5 \mathrm{~mm}$ were considered for the semi-quantitative evaluation, but the presence of smaller MCs was noted [13].

\section{Semi-quantitative measure of Modic changes}

From the scans showing at least one $\mathrm{MC}$, the unenhanced slice where each MC had the greatest depth was selected; the same slice was selected for the contrast-enhanced series. Unenhanced and contrastenhanced T1-weighted scans were co-registered to ensure alignment using Elastix, a registration toolkit based on the National Library of Medicine Insight Segmentation and Registration Toolkit (ITK) [19]. To this aim, two-dimensional affine registrations (six degrees of freedom) were performed.

In order to obtain semi-quantitative data, software allowing for manual selection of a polygonal region of interest (ROI) on the unenhanced image (Figs. 1 and 2) and pixel-based calculation of the signal intensity of the selected area was developed in Matlab (MathWorks, Natick, MA, USA).

For each MC under consideration, the developed protocol asked the operator to select a ROI corresponding to the zone of altered intensity on the unenhanced image and two ROIs as controls, because a reference for the "healthy" bone marrow in at least one control ROI 


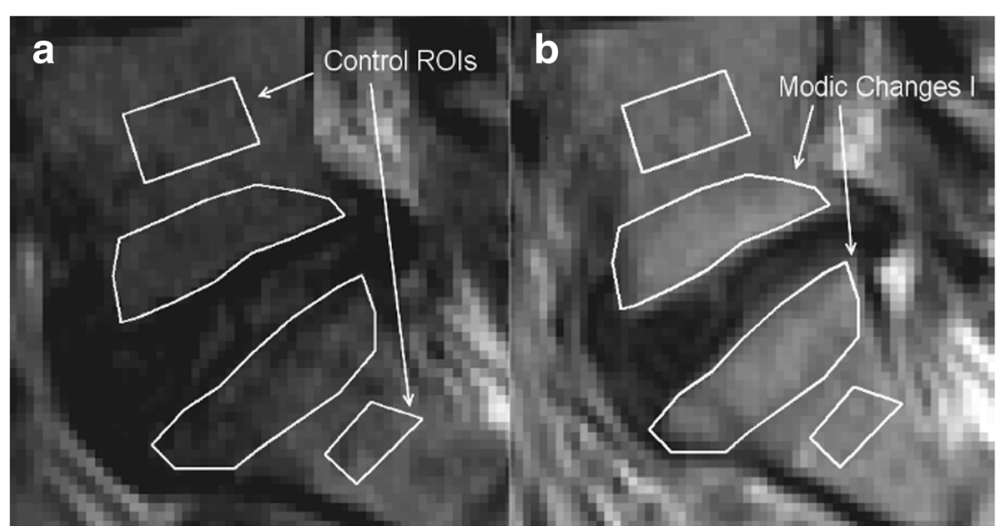

Fig. 1 Example of ROI selection for MCs I and same vertebra control areas on (a) unenhanced and (b) contrast-enhanced images. Two MCs I are present at the lower endplate of $L 5$ and the upper endplate of $\mathrm{S} 1 . \mathrm{RO}$ region of interest

was necessary. The ideal control ROI would be close enough to the MC to minimise the influence of local field fluctuation [20], but also wide enough and free of signal alteration, which in some cases would be possible only in another site. These considerations lead to an investigation of two different kinds of control ROI:

1. a ROI in the same vertebra affected with the MC (same vertebra $[\mathrm{SV}]$ );

2. a ROI corresponding to the section of the closest upper vertebra without $\mathrm{MC}$-in this case one ROI was used as reference for all the MCs in the same image (other vertebra $[\mathrm{OV}]$ ).

For each ROI, the following three indexes were calculated:

1. mean value of the pixels encompassed in the ROI (PRE):
$P R E=\operatorname{mean}\left(R O I_{p r e}\right)$

2. mean value of the difference between post and pre contrast signal intensity (DIFF):

$$
\mathrm{DIFF}=\text { mean }\left(\mathrm{ROI}_{\text {post }}-\mathrm{ROI}_{\mathrm{pre}}\right)
$$

3. ratio between PRE and DIFF, multiplied by 100 (NORM.DIFF):

$$
\text { NORM.DIFF }=\left(\frac{D I F F}{P R E}\right) * 100
$$

The three indexes calculated per ROI encompassing an $\mathrm{MC}$ were normalised with respect to the relevant control ROIs, to calculate the normalised signal intensity (NSI), as follows:

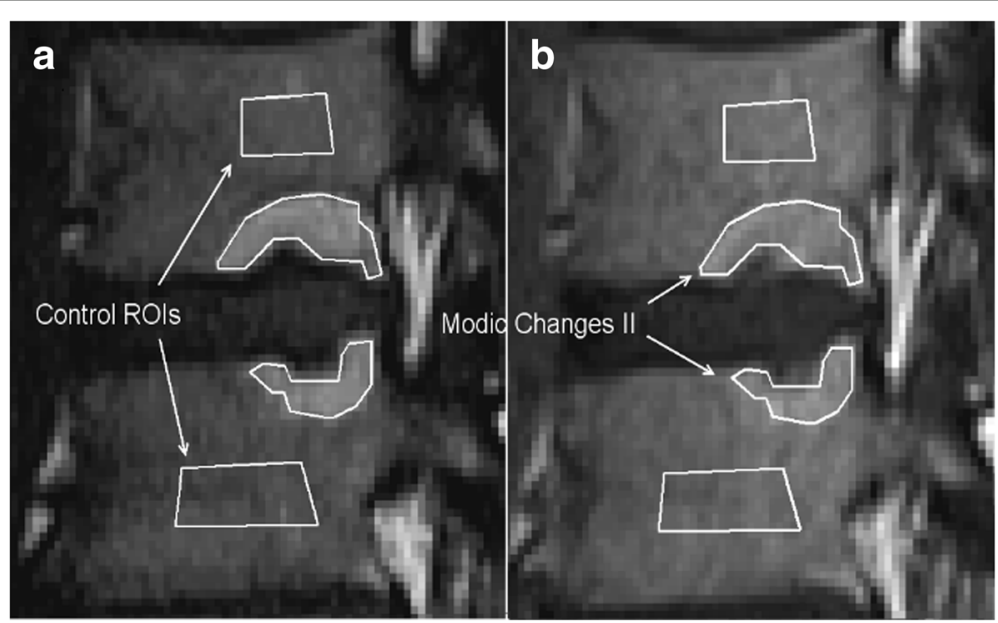

Fig. 2 Example of ROI selection for MCs II and same vertebra control areas on (a) unenhanced and (b) contrast-enhanced images. MCs II are present at the lower endplate of $L 1$ and the upper endplate of $L 2$, surrounding an endplate defect. $R O$ / region of interest 


$$
\mathrm{NSI}_{\mathrm{PRE}}=\frac{\left(\mathrm{PRE}_{\mathrm{MC}}-\mathrm{PRE}_{\mathrm{CONTROL}}\right)}{\mathrm{PRE}_{\mathrm{CONTROL}}} * 100
$$

NSI was extracted for PRE, DIFF and NORM.DIFF values and for each control ROI (SV, OV), for a total of six indexes.

In order to analyse inter-rater and intra-rater reliability, this procedure was repeated by the same operator 5 months after the first evaluation. A second operator, a resident in radiology with 3 years of experience not directly involved in the research, received a brief explanation about the software and the aim of the study before rating all data in one session.

\section{Statistical analysis}

Inter-rater and intra-rater agreement was analysed with the interclass correlation coefficient (ICC) (two-way mixed model, type absolute agreement), taking into consideration that an ICC of $0-0.2$ represents slight agreement, $0.21-0.4$ fair agreement, $0.41-0.6$ moderate agreement, $0.61-0.8$ substantial agreement and 0.81-1 excellent agreement [21].

The existence of significant differences in the described indexes among MC I, MC II and MC I/II was evaluated with a rank-sum test or Kruskal-Wallis one-way analysis of variance. The choice of a non-parametric test was justified by the non-normal distribution of data as confirmed by the Shapiro-Wilk test. If a statistical difference among groups was found, a multiple comparison procedure with Dunn's method was performed to establish the existence of difference among pairs. One-sample $t$ tests were carried out to test whether data had a mean significantly different with respect to zero. The presence of correlation between data was studied by Spearman rank-order correlation. Differences were considered significant when $p<0.050$.

\section{Results}

Among the 37 subjects enrolled in the study, 29 (78\%) were diagnosed as having at least one MC. Of the 444 considered endplates, 103 (23\%) had an MC and were classified as follows: 26 type I, 64 type II and 13 type I/ II. Sixty-one MCs with a height greater than $5 \mathrm{~mm}$ were then considered for the semi-quantitative analysis: 16 type I, 34 type II and 11 type I/II. MCs type III were absent in all patients.

\section{Intra-rater and inter-rater agreement}

Intra-rater agreement was excellent for all indexes considered (ICC 0.846-0.928). Inter-rater agreement was substantial or excellent (ICC 0.652-0.833). The type of control ROI did not influence reliability in any particular way.

\section{Control ROIs}

The choice of control ROI had no significant effect on the calculated indexes when all MCs were considered together (NSI ${ }_{\text {PRE }}, p=0.715 ; \mathrm{NSI}_{\text {DIFF }} p=0.539$; NSINORM.DIFF, $p=0.971$; rank-sum test). The same index calculated with respect to different control ROIs also showed high correlations: $R^{2}$ values calculated with Spearman correlation were 0.849 for $\mathrm{NSI}_{\mathrm{PRE}}, 0.79$ for $\mathrm{NSI}_{\text {DIFF }}$ and 0.72 for NSI NORM.DIFF $(p<0.001$ for all).

\section{Index for different MC types}

Figure 3 summarises the results of $\mathrm{NSI}_{\mathrm{PRE}}, \mathrm{NSI}_{\mathrm{DIFF}}$ and $\mathrm{NSI}_{\text {NORM.DIFF }}$ per MC type and control ROI. Every index reported a strong statistical difference $(p<0.001)$ among MC types and was able to discriminate between MC I and MC II. However, no index was able to discriminate between MCs I and MCs I/II. In particular, NSI $_{\text {PRE }}$ was higher than zero $(p<0.001)$ for MCs II, but lower than zero for MCs I $(p<0.001)$. For MCs I/II, NSI $_{\text {PRE }}$ was not statistically different from zero. MCs I and MCs I/II had a higher NSI DIFF than MCs II, and MCs I and MCs I/II had a median NSI DIFF higher than zero (all cases, $p<0.001$ ), while for MCs II only for SV control was the median value lower than zero $(p=0.029$; for OV, $p=0.213$ ). MCs I and MCs I/II had a higher $\mathrm{NSI}_{\text {NORM.DIFF }}$ than MCs II; the median NSI $\mathrm{NORM.DIFF}_{\text {for }}$ MCs I/II was lower than MCs I but the difference was not statistically significant. NSI $\mathrm{NORM.DIFF}_{\text {for MCs I and }}$ MCs I/II were higher than zero $(p<0.001$; but NSINORM.DIFF SV, $p=0.004)$, while MCs II had median values lower than zero $(p<0.001)$.

Fig. 4 shows scatter plots of $\mathrm{NSI}_{\mathrm{DIFF}}$ against $\mathrm{NSI}_{\mathrm{PRE}}$ and of $\mathrm{NSI}_{\text {NORM.DIFF }}$ against $\mathrm{NSI}_{\text {PRE }}$ for each control ROI. MCs I pool on the left of each graphic, because their PRE values are mostly negative, while MCs II pool on the right, with positive values. Considering enhancement indexes for $\mathrm{NSI}_{\text {DIFF }}$ and $\mathrm{NSI}_{\text {NORM.DIFF }}$ MCs I were characterised by positive values, while MCs II presented mostly negative values for $\mathrm{NSI}_{\text {NORM.DIFF }}$ (7 of 34 for $\mathrm{SV}$ and for $\mathrm{OV}$ were higher than zero) and were more scattered for $\mathrm{NSI}_{\text {DIFF }}(9 \mathrm{ROIs}$ report values higher than zero in SV, 14 for OV). MCs I/II always reported positive enhancement (apart from one case in $\mathrm{NSI}_{\text {NORM. }}$ DIFF SV, which is $-1 \%$ ).

\section{Discussion}

In this study we developed semi-quantitative indexes to analyse contrast enhancement of MCs with respect to "healthy" bone marrow on T1-weighted images. MCs I consistently showed a lower signal intensity compared with controls on unenhanced T1-weighted images as expected, and a marked enhancement on contrastenhanced T1-weighted images, significantly greater than the enhancement present in the "healthy" marrow. 

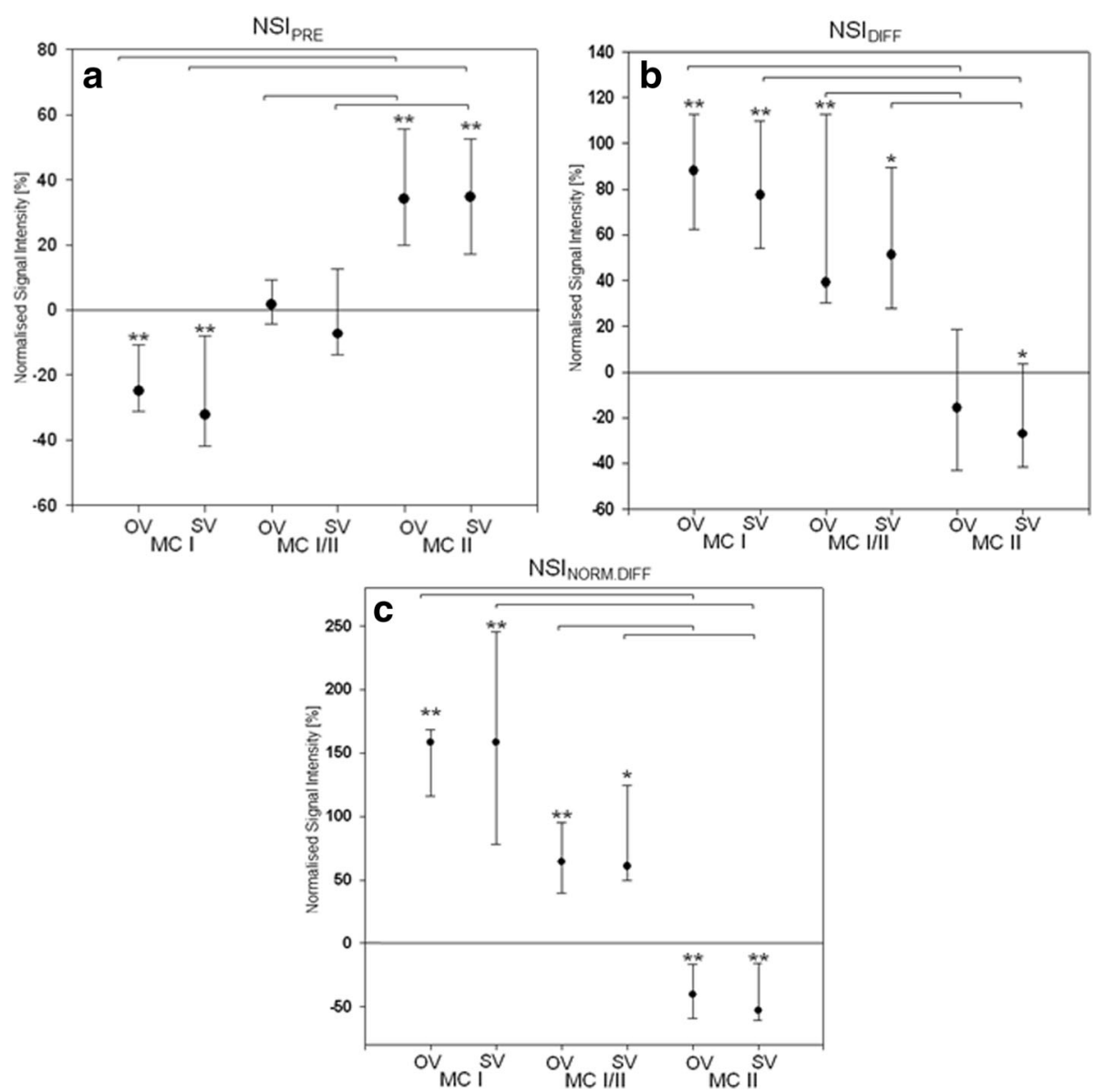

Fig. 3 Median and 25th and 75th percentiles of NSI PRE $(\mathbf{a}), \mathrm{NSI}_{\text {DIFF }}(\mathbf{b})$ and NSI NORM.DIFF $(\mathbf{c})$ for MC type and control ROI in the other vertebra (OV) or in the same vertebra (SV). ${ }^{*} p<0.050,{ }^{* *} p<0.001$ indicate whether the population has a median value significantly different with respect to zero. Bars indicate which pairs of MC type are significantly different (Dunn's method, $p<0.050)$. NSl PRE is higher than zero $(p<0.001)$ for MCs $\|$, is lower than zero $(p<0.001)$ for MCs I and is not statistically different from zero for MCs I/II. MCs I and MCs I/II have a higher NSIDIFF than MCs II, and MCs I and MCs I/II have a median NSI DIFF higher than zero (all cases, $p<0.001$ ), while for MCs II only for SV control was the median value lower than zero ( $p=0.029$; for OV, $p=0.213$ ). MCS I and MCs I/II have a higher NSI NORM.DIFF than MCs II; median NSINORM.DIFF for MCs I/II is lower than MCS I but is not statistically significant. NSI NORMDIFF median values for MCs I and MCs I/II are higher than zero $(p<0.001$; but NSI NORMDIFF SV, $p=0.004)$, while MCs II have median values lower than zero $(p<0.001)$. DIFF mean difference between post and pre contrast signal intensity, MC Modic change, NORM.DIFF ratio between PRE and DIFF $(\times 100)$, NSI normalised signal intensity, PRE mean pixels encompassed in the ROI

Conversely, MCs II showed a higher signal intensity on unenhanced T1-weighted images and a decreased or comparable enhancement with respect to controls. MCs I/II presented a behaviour generally intermediate between MCs I and MCs II, with a signal intensity on unenhanced T1-weighted images no different compared with the healthy marrow because higher and lower intensity zones tend to compensate for each other, and an enhancement close to MC I (see Figs. 3 and 4).

Considering the contrast enhancement as an indication of the grade of tissue vascularisation, these results are consistent with the suggestion that MCs I represent an inflammatory condition characterised by oedema and an augmented presence of small capillaries, while MCs II represent the substitution of haematopoietic marrow with fatty marrow, which possesses a sparse vascularity $[1,3]$.
We found that intra-rater agreement for the indexes considered was excellent, despite a time interval of 5 months between the two evaluations. Inter-rater agreement ranged between substantial and excellent, despite only providing a brief explanatory session to the resident before rating. These results are in agreement with the data on reliability on semi-quantitative measure of $\mathrm{MC}$ recently published by Wang et al. [13].

Wang et al. [13] speculated that the signal intensity of MCs on unenhanced T1-weighted images may indicate the severity of the degeneration. We verified that the contrast enhancement may add useful information about the degree of vascularisation in MCs, which strongly varies among types.

Moreover, contrast enhancement may help in the classification of mixed-type I/II MCs, which is more difficult 


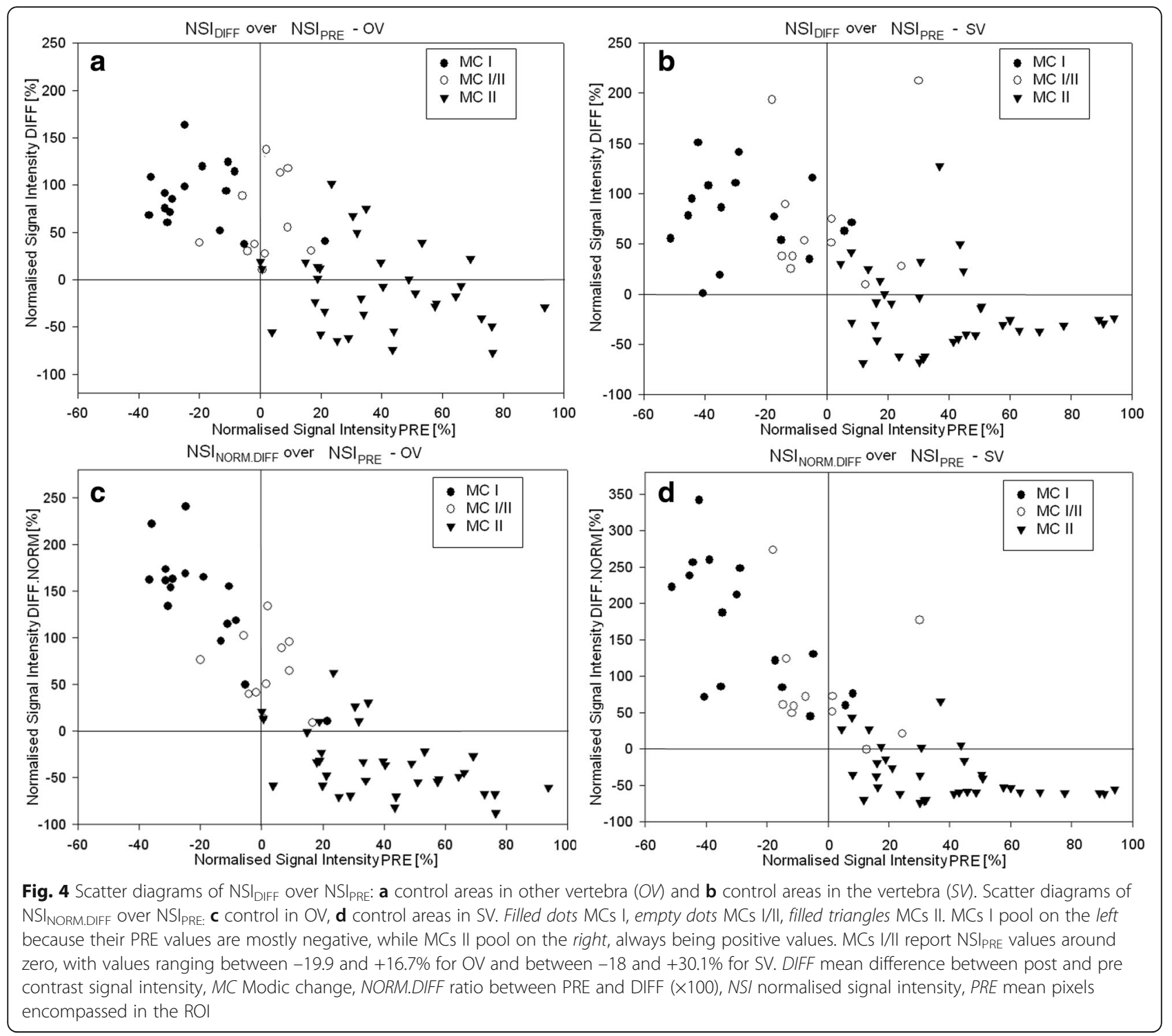

and requires more skills than the classification of pure type I or type II MCs [22]. The mixed type may represent an intermediate stage in a process of conversion between pure categories of MCs [3], which may be connected with the progression of the pathologic process [23]. Thus, a reliable classification based on contrast enhancement may help in the study of temporal evolution of MCs and of the degenerative disorder during patient follow-up, and eventually help in the clarification of this process, whose origin and dynamics are yet to be completely understood.

We have evaluated the simple and normalised difference between unenhanced and contrast-enhanced images. The pure, non-normalised difference demonstrated a change in the contrast enhancement in different MCs with respect to controls. The normalised difference has the advantage of being able to incorporate information about unenhanced signal intensity and the contrast enhancement, and can thus be used as a reference measure. Normalisation tends to give more sparse results than difference, particularly for MCs I, due to division of low values.

Given the lack of reliable reference for T1-weighted images, it was necessary to identify a ROI unaffected by $\mathrm{MC}$ as a control. If possible, we selected a seemingly "healthy" area in the same vertebral body affected by the degeneration, thus minimising magnetic field alterations [19]. Alternatively, in the case of an extensive MC involving most of the vertebral body, we selected an area in the vertebral body of the upper vertebra not affected with MC. We did not observe a significant difference in the indexes calculated with different control ROIs and also found a high correlation between indexes extracted with different controls, suggesting that the 
choice of the control ROI only has a marginal influence on the results.

The first limitation of this study is the low number of MCs considered, due to the relatively low number of subjects enrolled. Other studies based on a wider population would be necessary to verify the possibility of establishing reference values of contrast enhancement for MCs, to investigate whether the indexes described may be an indicator of the severity grade of the degenerative process as indicated by clinical indexes. Indeed, besides contributing to the basic knowledge about contrast-enhanced MRI of the spine, the availability of a semi-quantitative index could be useful to evaluate the progression of the degenerative disorder. Given the risk associated with the injection of gadolinium chelates $[24,25]$, we recognise that the simple investigation of MCs cannot be considered a justification for a contrast-enhanced MRI of the spine. Nevertheless, because this examination is performed for specific purposes in patients with previous history of spine surgeries or for other conditions such as fever, immunosuppression or oncological history, data on contrast enhancement of MCs may be available for a non-negligible number of patients. Second, we included only MCs with a height greater than $5 \mathrm{~mm}$, although an investigation of smaller MCs would be valuable for understanding the initiation mechanisms of endplate defects. This choice was related to the low spatial resolution of the images and the use of a subjective, manual method for the ROI creation, preventing an accurate and repeatable selection of the few pixels with altered signal intensity of small MCs. Third, T2-weighted fat-saturated images, commonly used to assess oedematous changes in MCs I [26], were not acquired in this study. Fourth, we did not investigate the possible correlation between MC enhancement and clinical data such as pain or quality of life scores, which were not collected in the primary study [17]. Finally, the study was conducted on a single 1.5-T scanner, and the findings are therefore to be considered valid only at that field strength.

In conclusion, based on the present results we do not recommend contrast injection for the MRI assessment of MCs alone, because they can be diagnosed qualitatively without contrast injection. However, the novel data reported interestingly show the difference in signal intensity of MCs on unenhanced and contrast-enhanced T1weighted images with respect to "healthy" bone marrow: MCs I consistently showed lower signal intensity than control areas on unenhanced images and marked contrast enhancement; MCs II showed a higher signal intensity on unenhanced images and a decreased or comparable contrast enhancement when compared with control areas; and MCs I/II presented a behaviour generally intermediate between MCs I and MCs II (unenhanced signal intensity close to MCs II and contrast enhancement close to MCs I).

\section{Funding}

The work was funded by the Seventh Framework Programme of the European Commission (grant number HEALTH-F2-2008-201626).

\section{Authors' contributions}

MT drafted the paper, performed statistical analysis and data processing, and participated to the data interpretation. CC participated to the study design and performed data collection and processing. VB participated to the statistical analysis. IMC performed the image analysis and participated to the data interpretation. $J U$ and MBB contributed to the study design and the data interpretation. FG contributed to the data interpretation and critically revised the paper draft. All authors read and approved the final manuscript.

\section{Competing interests}

The authors declare that they have no competing interests.

\section{Publisher's Note}

Springer Nature remains neutral with regard to jurisdictional claims in published maps and institutional affiliations.

\section{Author details}

${ }^{1}$ Department of Internal Medicine II-Cardiology, Ulm University, Helmholtzstrasse 16, 89081 Ulm, Germany. ${ }^{2}$ IRCCS Galeazzi Orthopedic Institute, Via Galeazzi 4, 20161 Milan, Italy. ${ }^{3}$ IRCCS Policlinico San Donato, Piazza Edmondo Malan 1, 20097 San Donato Milanese, Italy. ${ }^{4}$ Keele University, Keele ST5 5BG, UK. ${ }^{5}$ Department of Physiology, Anatomy and Genetics, Oxford University, Oxford OX1 3PA, UK. ${ }^{6}$ Department of Spine Surgery III, IRCCS Galeazzi Orthopaedic Institute, Via Galeazzi 4, 20161 Milan, Italy.

Received: 17 January 2017 Accepted: 28 March 2017

Published online: 29 June 2017

\section{References}

1. Modic M, Masaryk T, Ross J, Carter J (1988) Imaging of degenerative disk disease. Radiology 168:177-186

2. Modic M, Steinberg P, Ross J, Masaryk T, Carter J (1988) Degenerative disk disease: Assessment of changes in vertebral body marrow with MR imaging. Radiology 166:193-199

3. Braithwaite I, White J, Saifuddin A, Renton P, Taylor B (1998) Vertebral end-plate (Modic) changes on lumbar spine MRl: correlation with pain reproduction at lumbar discography. Eur Spine J 7:363-368

4. Z Zhang YH, Zhao CQ, Jiang LS, Chen XD, Dai LY (2008) Modic changes: a systematic review of the literature. Eur Spine J 17:1289-1299

5. Vogler J III, Murphy W (1988) Bone marrow imaging. Radiology 168:679-693

6. Kerttula L, Luoma K, Vehmas T, Grönblad M, Kääpä E (2012) Modic type I change may predict rapid progressive, deforming disc degeneration: a prospective 1-year follow-up study. Eur Spine J 21:1135-1142

7. Kuisma M, Karppinen J, Niinimäki J et al (2006) A three-year follow-up of lumbar spine endplate (Modic) changes. Spine (Phila Pa 1976) 31:1714-1718

8. Jensen TS, Karppinen J, Sorensen JS, Niinimäki J, Leboeuf-Yde C (2008) Vertebral endplate signal changes (modic change): a systematic literature review of prevalence and association with non-specific low back pain. Eur Spine J 17:1407-1422

9. Hutton MJ, Bayer JH, Powell JM (2011) Modic vertebral body changes: the natural history as assessed by consecutive magnetic resonance imaging. Spine 36:2304

10. Rahme R, Moussa R, Bou-Nassif R et al (2010) What happens to modic changes following lumbar discectomy? Analysis of a cohort of 41 patients with a 3-to 5-year follow-up period. J Neurosurg Spine 13:562-567

11. Barth M, Weiss C, Thomé C (2008) Two-year outcome after lumbar microdiscectomy versus microscopic sequestrectomy: Part 1: evaluation of clinical outcome. Spine 33:265-272

12. Jensen MC, Brant-Zawadzki MN, Obuchowski N et al (1994) Magnetic resonance imaging of the lumbar spine in people without back pain. $\mathrm{N}$ Engl J Med 331:69-73

13. Wang Y, Videman T, Niemeläinen R, Battié MC (2011) Quantitative measures of modic changes in lumbar spine magnetic resonance imaging: intra-and inter-rater reliability. Spine 36:1236-1243 
14. Kuisma M, Karppinen J, Haapea M et al (2009) Modic changes in vertebral endplates: a comparison of MR imaging and multislice CT. Skeletal Radiol 38:141-147

15. Boos N (2008) Spinal disorders: fundamentals of diagnosis and treatment. Springer Verlag, Heidelberg

16. Rosen BR, Belliveau JW, Vevea JM, Brady TJ (1990) Perfusion imaging with NMR contrast agents. Magn Reson Med 14:249-265

17. Tibiletti M, Galbusera F, Ciavarro C, Brayda-Bruno M (2013) Is the transport of a gadolinium-based contrast agent decreased in a degenerated or aged disc? A post contrast MRI study. PLoS One 8:e76697

18. Rajasekaran S, Babu JN, Arun R et al (2004) ISSLS prize winner: A study of diffusion in human lumbar discs: a serial magnetic resonance imaging study documenting the influence of the endplate on diffusion in normal and degenerate discs. Spine 29:2654-2667

19. Klein S, Staring M, Murphy K, Viergever MA, Pluim JPW (2010) Elastix: a toolbox for intensity-based medical image registration. IEEE Trans Med Imaging 29:196-205

20. Luoma E, Raininko R, Nummi P et al (1997) Suitability of cerebrospinal fluid as a signal-intensity reference on MRl: evaluation of signal-intensity variations in the lumbosacral dural sac. Neuroradiology 39:728-732

21. Shrout PE, Fleiss JL (1979) Intraclass correlations: uses in assessing rater reliability. Psychol Bull 86:420-428

22. Fayad F, Lefevre-Colau MM, Drapé JL et al (2009) Reliability of a modified modic classification of bone marrow changes in lumbar spine MRI. Joint Bone Spine 76:286-289

23. Kääpä E, Luoma K, Pitkäniemi J, Kerttula L, Grönblad M (2012) Correlation of size and type of modic types 1 and 2 lesions with clinical symptoms: a descriptive study in a subgroup of patients with chronic low back pain on the basis of a university hospital patient sample. Spine 37:134

24. Dillman JR, Ellis JH, Cohan RH, Strouse PJ, Jan SC (2007) Frequency and severity of acute allergic-like reactions to gadolinium-containing iv contrast media in children and adults. Am J Roentgenol 189:1533-1538

25. Lauenstein TC, Salman K, Morreira R et al (2007) Nephrogenic systemic fibrosis: center case review. J Magn Reson Imaging 26:1198-1203

26. Xu L, Chu B, Feng Y, Xu F, Zou YF (2016) Modic changes in lumbar spine: prevalence and distribution patterns of end plate oedema and end plate sclerosis. Br J Radiol 89:20150650

\section{Submit your manuscript to a SpringerOpen ${ }^{\circ}$ journal and benefit from:}

- Convenient online submission

- Rigorous peer review

- Immediate publication on acceptance

- Open access: articles freely available online

- High visibility within the field

- Retaining the copyright to your article

Submit your next manuscript at $>$ springeropen.com 